\title{
Use of Antipsychotic Drugs by Elderly Primary Care Patients and the Effects of Medication Reviews: A Cross-Sectional Study in Sweden
}

\author{
Cecilia Lenander ${ }^{1,2}(\mathbb{D}) \cdot$ Patrik Midlö $^{1} \cdot$ Nina Viberg ${ }^{1,3} \cdot$ John Chalmers $^{4}$. \\ Kris Rogers ${ }^{4}$ Åsa Bondesson ${ }^{1,2}$
}

Published online: 16 June 2017

(C) The Author(s) 2017. This article is an open access publication

\begin{abstract}
Background Antipsychotics form a class of drugs that should be used with caution among elderly people because of a high risk of adverse events. Despite the risks and modest effects, their use is estimated to be high, especially in nursing homes.

Objective The aim was to explore the effects of medication reviews on antipsychotic drug use for elderly primary care patients and describe the extent of, and reasons for, the prescription of antipsychotics.

Methods In this cross-sectional study in primary care in Skåne, Sweden, patients aged $\geq 75$ years living in nursing homes or in their own homes with home care were included. The effects of medication reviews were documented, as were the use of antipsychotics and the differences in characteristics between patients receiving or not receiving antipsychotics.

Results A total of 1683 patients aged $87.6( \pm 5.7)$ years were included in the analysis. Medication reviews reduced the use of antipsychotics by $23 \%(p<0.001)$ in this study. Of the 206 patients using antipsychotics, $43 \%(n=93)$ had an approved indication, while for $15 \%(n=32)$ the indication was not given. Antipsychotic drug use was more
\end{abstract}

Cecilia Lenander

cecilia.lenander@med.lu.se

1 Department of Clinical Sciences in Malmö, Lund University, Jan Waldenströms gata 35, 20502 Malmö, Sweden

2 Department of Medicines Management and Informatics, Region Skåne, Kristianstad, Sweden

3 Department of Public Health Sciences, Karolinska Institutet, Solna, Sweden

4 The George Institute for Global Health, University of Sydney, Sydney, Australia common with increasing number of drugs ( $p=0.001$ ), and in nursing home residents $(p<0.01)$. It was also more frequent in patients with cognitive impairment, depressive symptoms or sleeping problems.

Conclusion The use of antipsychotic drugs is high in elderly patients in nursing homes. They are often given for indications that are not officially approved or are poorly documented. Medication reviews appear to offer one useful strategy for reducing excessive use of these drugs.

\section{Key Points}

Potentially inappropriate antipsychotic drug use is high among elderly patients in nursing homes.

The use correlates with age and number of drugs.

Medication reviews reduced antipsychotic drug use by $23 \%$ in this study and could be one way of addressing the problem.

\section{Introduction}

In 2010, the Swedish National Board of Health and Welfare published a report with quality indicators concerning the treatment of elderly patients [1]. The report points to antipsychotics as a medication group that should be used with caution among elderly people. Prescription of antipsychotics should be limited to patients with psychotic events and potentially to aggressive patients with dementia according to the Swedish National Board of Health and 
Welfare [1]. Antipsychotic drugs carry a high risk of adverse events, such as extra-pyramidal symptoms, cognitive impairment, sedation and orthostatic hypotension as well as an increased risk of stroke and premature death in patients with dementia [2]. The use of antipsychotics for treatment of behavioural and psychological symptoms in dementia (BPSD) is still high. This is despite warnings from, for example, the US Food and Drug Administration (FDA), concerning the use of antipsychotics in patients with dementia [3]. Moreover, the evidence for the efficacy of antipsychotics for treating BPSD is at best modest for aggression and very weak for other symptoms [4].

Prescription of antipsychotics for elderly patients with multiple co-morbidities seems to be high and often for indications that are not officially approved. A Swedish study from 2004 [5], supported by the findings from other studies [2, 3, 6, 7], reported that $25-30 \%$ of patients in nursing homes were given antipsychotics, often without a clear indication.

Medication reviews provide a possible strategy to improve the situation. A medication review is a method to analyse, review and follow-up an individual's drug therapy. The review is done in a structured and systematic way, according to local guidelines and routines [8], and can be performed in hospitals, in primary care or in nursing homes. Medication reviews can be discussed at a multiprofessional meeting or left as a note to the doctor, with or without patient participation. Medication reviews should, according to the Swedish National Board of Health and Welfare, be performed in patients aged $\geq 75$ years who have five or more medications. Furthermore, for patients moving to a nursing home, medication reviews should be performed in connection with this and thereafter annually [9]. The aim of the present study was to assess the effects of medication reviews on antipsychotic drug use in elderly patients. We also wished to explore the extent of, and justification for, the prescription of antipsychotics for elderly patients in everyday practice and whether there were significant differences between patients receiving antipsychotics and those who did not receive treatment with antipsychotics.

\section{Method}

This was a cross-sectional study to examine the use of antipsychotics in elderly patients listed at private primary healthcare centres in Skåne, Sweden, and also to see if medication reviews could have an effect on antipsychotic drug use. It was based on everyday clinical practice in primary care with regular general practitioners (GPs), nurses and clinical pharmacists.

\subsection{Setting}

Almost all patients in Sweden are registered with a GP as their primary care provider. Primary care in Skåne (a region in southern Sweden) is provided by public or private primary care centres, which are all funded by the county council and financed by taxes. At the time of the study, there were 151 primary care centres in Skåne and $43 \%$ of these were private. During 2011-2012, the private primary care centres in Skåne were offered medication reviews guided by a clinical pharmacist. A total of 25 out of 65 centres accepted. Seven clinical pharmacists were involved in the medication reviews, and they all had at least 3 years' experience in performing medication reviews. Patients aged 75 years or older living in nursing homes or their own homes with municipally provided home care who received a medication review were included in this study. For patients who had more than one medication review, only the first one was included in the analysis.

\subsection{Medication Reviews According to the LIMM Model}

The Lund Integrated Medication Management (LIMM) model has been described previously $[10,11]$; therefore, the principal features are only briefly outlined here. A nurse did a symptom assessment using PHASE-20 (Pharmacotherapeutical Symptom Evaluation, 20 questions) [12], including medical information such as current diagnosis, blood pressure, pulse, weight (measured by the nurse) and creatinine levels, as well as questions about symptoms such as dizziness, cognitive impairment and anxiety. For patients who were not able to answer these questions, the nurse would get assistance from other nursing staff. PHASE-20 is a validated tool for use in connection with medication reviews for identifying possible drug-related symptoms in older people [12]. The tool has been recommended for use in medication reviews by the Swedish National Board of Health and Welfare [1]. The PHASE-20 was sent together with a medication list to the pharmacist. The pharmacist used this information to identify drug-related problems (DRPs) and suggest recommendations to overcome these problems. All this was then discussed at a team meeting with the GP caring for the patient, the pharmacist, the nurse and in some cases other nursing staff. Based on the discussion, notes in the medical record and his/her clinical knowledge, the GP then decided on appropriate measures. The results of the changes were followed up (better, worse, unchanged) at 4-8 weeks by the nurse and forwarded to the pharmacist.

All data were collected in an Access database. Patient anonymity was accomplished by using a non-traceable ID number. 


\subsection{Data Collection}

For all patients, age, gender, type of housing (nursing home or ordinary home with home care), number of medications, use of antipsychotics, indications for this and symptoms (from PHASE-20) were recorded. As this was a retrospective analysis, the information available was from the medication review/team meeting, i.e. symptom assessment form, medication list and suggested recommendations from the pharmacist. We had no access to the medical record during the analysis.

We identified all patients who received antipsychotics by reviewing the medication lists. Antipsychotics in this study included the following Anatomical Therapeutic Chemical Classification System (ATC) [13] groups: all drugs in N05A, except for lithium, and R06AD01 (alimemazine). For all patients treated with antipsychotics, the reason/indication was identified using the symptom assessment form, the medication list and the list of suggested recommendations from the pharmacist (which included notes from the discussion at the team meeting). A judgement as to whether the reason/indication was appropriate or not was then made on the basis of this information. According to the Swedish quality indicators [1], the following indications were regarded as appropriate: psychotic conditions, other disease with psychotic symptoms, and dementia with BPSD such as severe aggressiveness. All other indications were regarded as potentially inappropriate. For patients with Lewy body dementia, all indications were regarded as inappropriate. If no reason/ indication could be found, this was registered.

We also noted whether the use of antipsychotics was discussed during the medication review and whether any changes to therapy were made. Only medication changes that were decided upon during the medication review meeting were included in the study measures.

\subsection{Data Analysis}

Descriptive analysis included average age and sex distribution of the patients, number of drugs, type of living, use of antipsychotics and the type of antipsychotic drugs used. Percentage of patients with an appropriate indication for antipsychotics was analysed and for what indications. Distribution of types of DRPs (according to Cipolle et al. [8]) suggested intervention recommendations, and the related treatment adjustments were also determined.

Data were analysed using IBM SPSS version 23 [14]. Ordinal variables from the PHASE-20 representing symptom severity were dichotomised. Symptoms were considered present if they were rated as moderate or severe, and absent if they were rated as none or small in the original data. Differences in proportion of symptoms between patients who were/were not prescribed antipsychotics were tested using a $\chi^{2}$ test on a two-way table. Resident characteristics were assessed for their association with prevalent antipsychotic use in univariable logistic regression models. Variables with $p$ values under 0.20 were used in an adjusted multivariable logistic regression model, and we used the likelihood ratio test to produce $p$ values.

The analyses were based on antipsychotic use prior to the medication review. A significance level of 0.05 was used.

\section{Results}

A total of 1683 patients were included in the analysis, and of these, $12 \%(n=206)$ used antipsychotics. The patients included had a mean age of 87.6 years and on average used 10.9 drugs (range 1-29). Polypharmacy was frequent, with 95\% using five or more drugs and 58\% using ten or more drugs. A majority of the patients were females and lived in nursing homes (Table 1).

Of the 206 patients using antipsychotics, only $43 \%$ $(n=93)$ had an approved indication, while for $15 \%$ $(n=32)$, no indication was given (Table 2). The most common indications recorded were psychosis or psychotic symptoms $(n=80)$, anxiety $(n=61)$, none specified $(n=32)$ and dementia $(n=19)$. Of the patients on antipsychotics, $66.5 \%$ of those in nursing homes had used the antipsychotic drug for more than 3 months, compared with $50 \%$ in the home care group.

The antipsychotics most commonly used were risperidone $(n=92)$, haloperidol $(n=45)$, olanzapine $(n=26)$ and alimemazine $(n=21)$.

Before performing the medication review, 206 patients used antipsychotics: 198 in nursing homes and eight in home care. Nine patients were prescribed two antipsychotic drugs at the same time. After the medication review, this had decreased to 157 patients (151 in nursing home, six in home care), i.e. a decrease of $23 \%(p<0.001)$. For two of the patients receiving two antipsychotic drugs, one of the drugs was withdrawn following the medication review. No antipsychotic medication was prescribed as a result of the medication review. The most common indications after the medication review were the same as before: psychosis or psychotic symptoms $(n=63)$, anxiety $(n=50)$, none specified $(n=23)$ and dementia $(n=11)$. The largest decrease was seen for psychosis and psychotic symptoms $(-21 \%)$.

During the medication review, the pharmacist brought up the treatment with antipsychotics as a problem for all patients in home care and in $80 \%$ of the cases in nursing homes. The most common DRPs were wrong drug (60\%), unnecessary drug therapy (18\%) and adverse events (16\%). 
Table 1 Baseline characteristics

\begin{tabular}{|c|c|c|c|c|c|c|c|}
\hline \multirow[t]{2}{*}{ Characteristic } & \multicolumn{2}{|c|}{ Antipsychotic drug use } & \multirow[t]{2}{*}{ Overall } & \multicolumn{2}{|l|}{ Unadjusted } & \multicolumn{2}{|l|}{ Adjusted $^{\mathrm{a}}$} \\
\hline & $\begin{array}{l}\text { Yes } \\
n=206\end{array}$ & $\begin{array}{l}\text { No } \\
n=1477\end{array}$ & & OR $(95 \% \mathrm{CI})$ & $p$ value & OR $(95 \% \mathrm{CI})$ & $p$ value \\
\hline Female, $n(\%)$ & $140(68.0)$ & $1080(73.1)$ & $1220(72.5)$ & $1.29(0.94-1.77)$ & 0.11 & $0.80(0.60-1.11)$ & 0.17 \\
\hline Age (years), mean (SD) & $86.3(5.9)$ & $87.8(5.7)$ & $87.6(5.7)$ & $0.96(0.94-0.98)$ & 0.001 & $0.96(0.94-0.99)$ & 0.02 \\
\hline Nursing home residents, $n(\%)$ & $198(96.1)$ & $1276(86.4)$ & $1474(87.6)$ & $0.26(0.13-0.53)$ & $<0.001$ & $4.20(2.03-8.67)$ & $<0.0001$ \\
\hline $\begin{array}{l}\text { Number of drugs per patient (except } \\
\text { antipsychotics), mean (SD) }\end{array}$ & $12.2(4.6)$ & $10.9(4.5)$ & $10.9(4.5)$ & $1.01(0.98-1.04)$ & 0.56 & $1.06(1.03-1.10)$ & 0.001 \\
\hline
\end{tabular}

$C I$ confidence interval, $O R$ odds ratio, $S D$ standard deviation

${ }^{\text {a }}$ Nagelkerke $R^{2}=0.05$, Hosmer-Lemeshow goodness-of-fit $p=0.58$

Table 2 Indications for use of antipsychotics $(n=215$, with 9 patients having 2 antipsychotics, i.e. 206 patients)

\begin{tabular}{lcllll}
\hline Indications & \multicolumn{2}{l}{ Before medication review } & & \multicolumn{2}{l}{ After medication review } \\
\cline { 2 - 3 } \cline { 5 - 6 } & $n(\%)$ & Approved indication $^{\mathrm{a}}$ & & $n(\%)$ & Approved indication $^{\mathrm{a}}$ \\
\hline Psychosis or psychotics symptoms & $80(37)$ & $77^{\mathrm{b}}$ & $63(38)$ & $61^{\mathrm{b}}$ \\
BPSD & $11(5)$ & 11 & $8(5)$ & 8 \\
Nausea & $4(2)$ & - & $2(1)$ & - \\
Anxiety & $61(28)$ & - & $50(30)$ & - \\
Depression & $1(0.5)$ & - & $1(0.6)$ & - \\
Dementia & $19(9)$ & - & $11(7)$ & - \\
Bipolar disease & $6(3)$ & $5^{\mathrm{c}}$ & $6(4)$ & $5^{\mathrm{c}}$ \\
Sleep & $1(0.5)$ & - & - & - \\
None specified & $32(15)$ & - & $23(14)$ & - \\
\hline
\end{tabular}

$B P S D$ behavioural and psychological symptoms in dementia

a According to Swedish National Board of Health and Welfare (2010) (indicators for the evaluation of quality in drug use of the elderly)

b Three patients before and 2 patients after medication review with Lewy body dementia treated with antipsychotics for psychotic symptoms

c Olanzapine approved
The most common advice offered was to evaluate the efficacy and possible adverse effects of the drug therapy (48\%), followed by withdrawal of drug (19\%) and progressive lowering of dose (19\%). This advice was acted upon by the doctor in about one quarter of the cases, both in nursing homes and home care. After medication review, 84 patients with an inappropriate indication or no indication for antipsychotics still used them.

The use of antipsychotics among patients in nursing homes was more common (13.4\%) than in patients with home care $(3.8 \%)(p<0.01)$. There was a nonsignificant difference between the sexes in the use of antipsychotics: men $14.3 \%$ compared with women $11.4 \%(p=0.11)$. Among patients with antipsychotics, the use of ten or more drugs was more common $(65.5 \%)$ than in patients without $(57 \%)(p=0.02)$. Fewer of the patients using antipsychotics could participate (fully or partly) in answering the symptom assessment compared with those not using antipsychotics $(43.0$ vs. $57 \%, p<0.001)$. In the group receiving antipsychotics, cognitive impairment, depressive symptoms, anxiety and feeling tired were more common in the symptom assessment than among those not on antipsychotics (Table 3).

Older patients had lower odds of being prescribed antipsychotics [odds ratio $(\mathrm{OR})=0.96 ; 95 \%$ confidence interval $(\mathrm{CI})$ 0.94-0.99], while nursing home residents had higher odds (OR $4.20 ; 95 \%$ CI 2.03-8.67). Increased number of drugs was also associated with higher odds of being prescribed antipsychotics (OR 1.06; 95\% CI 1.03-1.10) (Table 1).

\section{Discussion}

Our study shows that medication reviews offer a useful strategy for reducing excessive use of antipsychotic drugs. We found that the use of antipsychotics in nursing homes is 
Table 3 Symptoms from PHASE-20 assessment and their frequencies in patients with or without antipsychotic drugs

\begin{tabular}{lllc}
\hline Symptoms & Antipsychotic use $(n=206)(\%)$ & No antipsychotic $(\%)$ & $p$ value \\
\hline Sleeping problems & 24.9 & 18.6 & 0.025 \\
Depression & 41.0 & 27.6 & $<0.001$ \\
Fatigue & 60.0 & 50.5 & $<0.01$ \\
Cognitive impairment & 69.8 & 56.0 & $<0.001$ \\
Anxiety & 51.7 & 30.0 & $<0.001$ \\
Irritability & 41.0 & 27.6 & $<0.001$ \\
Dizziness & 37.1 & 36.3 & NS \\
Participation in answering symptom assessment & 43.0 & 57.0 & $<0.001$ \\
\hline
\end{tabular}

NS not significant, PHASE-20 Pharmacotherapeutical Symptom Evaluation, 20 questions

a Chi-square test

common, often without an approved indication and that in many cases the patients had been receiving treatment for more than 3 months. We also found that patients on antipsychotics more often reported symptoms such as anxiety, depressive symptoms and cognitive impairment. The use of antipsychotics decreased with age, but increased with nursing home residency and number of drugs.

In our study, $13 \%$ of patients in nursing homes had a prescription for antipsychotics. This is in line with other studies $[15,16]$. However, an Israeli study showed variations between 14.8 and $70.6 \%$ [17] depending on setting, and another Swedish study reported $38 \%$ of patients with dementia were on antipsychotic drugs [18].

According to a study by Kamble et al., one third of dementia patients in nursing homes receive antipsychotics; men more often than women [19]. Another study [15] reported that the use of antipsychotics was higher for women, patients with polypharmacy and those with decreased functional status. In our study, antipsychotic use was more prevalent in nursing home residents, in patients with many drugs and in younger patients. Around $57 \%$ of the patients using antipsychotics in this study did not receive the drug for an approved indication. This is lower than in a recent Canadian study, where $70-80 \%$ of the use was regarded as potentially inappropriate [20]. The most common drugs used were risperidone and haloperidol, which is in line with the recommendations for the use of antipsychotics in Sweden [1].

The FDA has issued a warning for increased morbidity and mortality in patients with dementia treated with antipsychotics [3]. However, antipsychotics are frequently used to treat psychiatric symptoms in patients with dementia [17, 21].

According to most guidelines on treatment of BPSD, the first step is to identify triggering factors (somatic problems, medications, environmental factors) and remove them. If this is not enough, non-pharmacological interventions, for example, a person-centred approach and distraction techniques, should be tried before using antipsychotics. In Sweden, many nursing homes work with the Swedish BPSD register [22] to improve the quality of care of patients with dementia. The register has a clear structure that relies on outlining the frequency and severity of BPSD using the Neuropsychiatric Inventory (NPI) scale, documenting current medications, and providing a checklist for possible causes of BPSD, and offers evidence-based care plan proposals to reduce BPSD as well as an evaluation of the interventions employed. According to the Swedish indicators [1], treatment effects of antipsychotics should be evaluated and attempts at lowering the dose should be undertaken within 2 weeks after initiation of therapy. Multi-dose drug dispensing (i.e. all drugs that are to be ingested at the same time are machine-dispensed in unit bags) is common at Swedish nursing homes. There are studies showing that multi-dose dispensing leads to increased treatment times in general [23], which could be one explanation for the higher rates of antipsychotic use in nursing homes. It is also known that patients with conditions such as severe BPSD cannot stay at home, but are instead moved into a nursing home [24]. This is in line with a French study [25] indicating that for patients with Alzheimer's disease, treatment with antipsychotics is more common in nursing homes than in home care.

In the majority of the medication reviews for patients with antipsychotic drugs, the use was discussed during the team meeting. Action was taken by the doctor in $25 \%$ of the cases, and the use of antipsychotics was decreased by $23 \%$. Compared with acceptance rates for pharmacist recommendations during medication reviews including all kinds of drugs, $25 \%$ is a small percentage [11, 26-28]. This could be due to the need for tapering antipsychotics slowly, or perhaps the doctors need more time to consider the 
suggestions before acting. There might also be cases where other changes had to be done first or circumstances where the doctor wants to wait, e.g. in the case of a newly moved patient. Since this was a study in everyday practice with no extra resources for follow-up, the only decisions recorded were those taken at the team meeting. It is possible, however, that the doctor acted upon the given advice sometime after the team meeting, considering the large proportion of patients with an inappropriate indication for antipsychotic drug use.

The study included a non-selected group of patients, with many patients and GPs from 25 different primary care centres, in the Skåne region. There were seven clinical pharmacists who participated in the team-based medication reviews. The study was done in everyday practice, reflecting the actual use of antipsychotics and the problems associated with that use. A symptom assessment was used as one source of information; this together with the GPs and the nurses' knowledge about the patients was used to decide on therapy changes. The use of a symptom assessment gives an overall picture of a patient's problems, which might make it easier to decide on, for example, discontinuation. All patients in our study were discussed at a team meeting, with the GP, the nurse and pharmacist present. According to the literature [29-31] face-to-face meetings are important for effective teamwork, for the establishment of trust in one another and, thereby, for achieving a higher acceptance rate for the pharmacist recommendations.

Our study has some limitations. Antipsychotics were not the only focus in the medication reviews, even though their use was discussed as a problem in the vast majority of the reviews. The participating doctors and pharmacists may have had different priorities. The medication reviews were conducted in everyday clinical practice, with limited resources, including the number of clinical pharmacists, so that not all patients at the included primary care centres received a medication review and follow-up information was not routinely collected. Nor did we have access to patient records during the analysis, and therefore we could not establish the connection between onset of symptoms and the timing of the prescription of antipsychotics. Furthermore, as this was a cross-sectional observational study, the findings are limited to reporting associations and cannot infer causality, for example, in relation to the link between the use of antipsychotics and the frequency of cognitive impairment, depressive symptoms and sleeping disorders. Neither could we consider all co-morbidities, since we did not have access to patient records during analysis. We also had a mixed population, i.e. both patients with a dementia diagnosis and patients without. However, the Swedish
National Board of Health and Welfare does not distinguish between the groups regarding treatment with antipsychotics [1], and it is estimated that around $70 \%$ of the nursing home residents in Sweden have some sort of cognitive impairment. We also looked at treatment with antipsychotics (yes/no), not at the doses used.

Future studies with improved follow-up are needed to evaluate the long-term effects of medication reviews on antipsychotic use as well as to establish the link between polypharmacy and antipsychotic drug use.

\section{Conclusion}

The use of antipsychotic drugs was high in this population of elderly patients in nursing homes in Sweden, particularly in those with cognitive impairment, depression or difficulty in communication. They are often prescribed for indications that are not officially approved or are poorly documented. Medication reviews appear to offer a useful strategy for reducing excessive use of these drugs, and should be explored in subsequent studies.

Acknowledgements The authors thank the pharmacists who performed the medication reviews (Nadia Al-Omar, Linda Andersson, Sara Andersson, Emma Brogård, Karolina Engvall and Emma Lundgren), and the participating municipal care nurses and GPs. We are indebted to Patrick Reilly and Karin Luton for their expertise and valuable advice in editing the manuscript.

Authors' Contributions CL and $\AA \mathrm{B}$ designed the study with input from PM. CL collected the data and conducted the analyses with help from KR. CL drafted the manuscript with help from PM and $\AA \mathrm{B}$. JC and NV made substantial contributions to the manuscript and its conclusions. All authors read, contributed to and approved the final manuscript.

\section{Compliance with ethical standards}

Conflict of interest CL, PM, NV, JC, KR and AB declare that they have no conflict of interest.

Ethical approval The study was approved by the Regional Ethical Review Board in Lund (application number 2013/649).

Funding This study was supported by a grant from the Swedish Society of Medicine, Gyllenstierna Krapperup foundation and Fromma foundation; by ALF funding from Region Skåne, awarded to PM; and by the Department of Medicines Management and Informatics, Region Skåne, Sweden.

Open Access This article is distributed under the terms of the Creative Commons Attribution-NonCommercial 4.0 International License (http://creativecommons.org/licenses/by-nc/4.0/), which permits any noncommercial use, distribution, and reproduction in any medium, provided you give appropriate credit to the original author(s) and the source, provide a link to the Creative Commons license, and indicate if changes were made. 


\section{References}

1. Swedish National Board of Health and Welfare. Indicators for the evaluation of quality in drug use of the elderly. Report no. 2010-6-29, Stockholm. 2010.

2. Schneider LS, Dagerman KS, Insel P. Risk of death with atypical antipsychotic drug treatment for dementia: meta-analysis of randomized placebo-controlled trials. JAMA. 2005;294(15): 1934-43.

3. US Food and Drug Administration. Information for healthcare professionals: conventional antipsychotics. 2008. http://www.fda. gov/Drugs/DrugSafety/PostmarketDrugSafetyInformationforPatient sandProviders/ucm 124830.htm.

4. Seitz DP, Gill SS, Herrmann N, Brisbin S, Rapoport MJ, Rines J, et al. Pharmacological treatments for neuropsychiatric symptoms of dementia in long-term care: a systematic review. Int Psychogeriatr IPA. 2013;25(2):185-203.

5. Swedish National Board of Health and Welfare. Quality of drug use in elderly patients [Kvaliteten i äldres läkemedelsanvändningKÄLLA-projektet]. Report no. 2004-131-24, Stockholm. 2004.

6. Wettermark B, Hammar N, Fored CM, Leimanis A, Otterblad Olausson P, Bergman U, et al. The new Swedish Prescribed Drug Register-opportunities for pharmacoepidemiological research and experience from the first six months. Pharmacoepidemiol Drug Saf. 2007;16(7):726-35.

7. Shah SM, Carey IM, Harris T, Dewilde S, Cook DG. Antipsychotic prescribing to older people living in care homes and the community in England and Wales. Int $\mathrm{J}$ Geriatr Psychiatry. 2011;26(4):423-34.

8. Cipolle RJ, Strand LM, Morley PC. Pharmaceutical care practice. New York: The McGraw-Hill Companies Inc; 1998.

9. Swedish National Board of Health and Welfare. SOSFS 2012:9. Stockholm 2012

10. Hellstrom LM, Bondesson A, Hoglund P, Midlov P, Holmdahl L, Rickhag E, et al. Impact of the Lund integrated medicines management (LIMM) model on medication appropriateness and drugrelated hospital revisits. Eur J Clin Pharmacol. 2011;67(7): $741-52$.

11. Milos V, Rekman E, Bondesson A, Eriksson T, Jakobsson U, Westerlund $\mathrm{T}$, et al. Improving the quality of pharmacotherapy in elderly primary care patients through medication reviews: a randomised controlled study. Drugs Aging. 2013;30(4):235-46.

12. Hedström M, Lidström B, Hulter-Åsberg K. PHASE-20: a new instrument for assessment of possible therapeutic drug-related symptoms among elderly in nursing homes [in Swedish]. Nordic J Nurs Res Clin Stud (Vård i Norden). 2009;4:9-14.

13. WHO. Anatomical Therapeutic Chemical Classification System [online]. http://www.who.int/classifications/atcddd/en. Accessed 4 Nov 2016.

14. IBM Corp. IBM SPSS Statistics for Windows, Version 22.0. Armonk, NY 2013.

15. Vidal X, Agusti A, Vallano A, Formiga F, Moyano AF, Garcia J, et al. Elderly patients treated with psychotropic medicines admitted to hospital: associated characteristics and inappropriate use. Eur J Clin Pharmacol. 2016;72(6):755-64.

16. The Swedish National Board of Health and Welfare. [Open comparisons drug use]. Stockholm 2014. Report No.: 2014-11-1.
17. Frankenthal D, Zandman-Goddard G, Ben-Muvhar Y, Porat-Katz BS. The impact of facility characteristics on the use of antipsychotic medications in nursing homes: a cross-sectional study. Isr J Health Policy Res. 2016;5:12.

18. Gustafsson M, Karlsson S, Lovheim H. Inappropriate long-term use of antipsychotic drugs is common among people with dementia living in specialized care units. BMC Pharmacol Toxicol. 2013;14:10.

19. Kamble P, Chen H, Sherer J, Aparasu RR. Antipsychotic drug use among elderly nursing home residents in the United States. Am J Geriatr Pharmacother. 2008;6(4):187-97.

20. Stock KJ, Amuah JE, Lapane KL, Hogan DB, Maxwell CJ. Prevalence of, and resident and facility characteristics associated with antipsychotic use in assisted living vs. long-term care facilities: a cross-sectional analysis from Alberta, Canada. Drugs Aging. 2017;34(1):39-53.

21. Ballard C, Howard R. Neuroleptic drugs in dementia: benefits and harm. Nat Rev Neurosci. 2006;7(6):492-500.

22. BPSD register. http://www.bpsd.se/other-languages/english/.

23. Sjoberg C, Ohlsson H, Wallerstedt SM. Association between multi-dose drug dispensing and drug treatment changes. Eur $\mathrm{J}$ Clin Pharmacol. 2012;68(7):1095-101.

24. Porter CN, Miller MC, Lane M, Cornman C, Sarsour K, KahleWrobleski K. The influence of caregivers and behavioral and psychological symptoms on nursing home placement of persons with Alzheimer's disease: a matched case-control study. SAGE Open Med. 2016;4:2050312116661877.

25. Jacquin-Piques A, Sacco G, Tavassoli N, Rouaud O, Bejot Y, Giroud M, et al. Psychotropic drug prescription in patients with dementia: nursing home residents versus patients living at home. J Alzheimers Dis. 2015;49(3):671-80.

26. Gheewala PA, Peterson GM, Curtain CM, Nishtala PS, Hannan PJ, Castelino RL. Impact of the pharmacist medication review services on drug-related problems and potentially inappropriate prescribing of renally cleared medications in residents of aged care facilities. Drugs Aging. 2014;31(11):825-35.

27. Finkers F, Maring JG, Boersma F, Taxis K. A study of medication reviews to identify drug-related problems of polypharmacy patients in the Dutch nursing home setting. J Clin Pharm Ther. 2007;32(5):469-76.

28. Brulhart MI, Wermeille JP. Multidisciplinary medication review: evaluation of a pharmaceutical care model for nursing homes. Int J Clin Pharm. 2011;33(3):549-57.

29. Frankenthal D, Israeli A, Caraco Y, Lerman Y, Kalendaryev E, Zandman-Goddard $\mathrm{G}$ et al. Long-term outcomes of medication intervention using the screening tool of older persons potentially inappropriate prescriptions screening tool to alert doctors to right treatment criteria. J Am Geriatr Soc. 2016;65(2):e33-e38

30. Chen TF, de Almeida Neto AC. Exploring elements of interprofessional collaboration between pharmacists and physicians in medication review. Pharm World Sci. 2007;29(6):574-6.

31. Kwint HF, Bermingham L, Faber A, Gussekloo J, Bouvy ML. The relationship between the extent of collaboration of general practitioners and pharmacists and the implementation of recommendations arising from medication review: a systematic review. Drugs Aging. 2013;30(2):91-102. 